\title{
Analisando Fatores que Afetam o Desempenho de Estudantes Iniciantes em um Curso a Distância
}

\author{
Jorge Luis Cavalcanti Ramos ${ }^{1,3}$, Rodrigo Lins Rodrigues ${ }^{2,3}$, João Carlos Sedraz \\ Silva ${ }^{1,3}$, Alex Sandro Gomes ${ }^{3}$ \\ ${ }^{1}$ Colegiado de Engenharia da Computação - Universidade Federal do Vale do São \\ Francisco-UNIVASF \\ ${ }^{2}$ Departamento de Educação - Universidade Federal Rural de Pernambuco - UFRPE \\ ${ }^{3}$ Centro de Informática - Universidade Federal de Pernambuco - UFPE \\ jorge.cavalcanti@univasf.edu.br, rlreded.ufrpe.br, \\ joão.sedraz@univasf.edu.br, asg@cin.ufpe.br
}

\begin{abstract}
This study aimed to identify some variables that were significantly related to the performance of beginner's students in a course in distance mode. The literature suggests several barriers to Distance Learning, among them difficulties in adapting itself to the modality, the skill in the use of technological resources that are required for day to day, among others. From the questionnaires at the beginning of a higher distance course and the crossing of some variables with the performance of students, the results indicated that prior experience with distance education, adequate knowledge in computer and easily adaptable to new technologies had significant relationship with student achievement in the first discipline of the course.
\end{abstract}

Resumo. Este estudo teve como objetivo identificar algumas variáveis que possuem relação significativa com o desempenho de alunos iniciantes em um curso na modalidade a distância. A literatura aponta várias barreiras à Educação a Distância (EAD), dentre as quais dificuldades relacionadas com a própria adaptação à modalidade, a habilidade na utilização de recursos tecnológicos que são necessários para o dia a dia, entre outras. A partir da aplicação de questionários no início de uma disciplina de curso superior a distância e o cruzamento de algumas variáveis com o desempenho dos alunos, os resultados apontaram que a experiência prévia com EAD, conhecimento satisfatório em informática e facilidade de adaptação a novas tecnologias tiveram relação significativa com o desempenho do aluno na primeira disciplina do curso.

\section{Introdução}

Com o crescimento acentuado nos últimos anos, a Educação a Distância (EAD) tem assumido um importante papel no cenário educacional, em particular na formação em nível superior de setores da população que dificilmente alcançariam essa categoria de escolaridade, por fatores sociais e econômicos diversos. Essa modalidade viabiliza a formação em horário flexível distinto dos horários em ambientes escolares presenciais, possui um alcance geográfico muito abrangente, assim como possibilita redução de custos na formação profissional do cidadão. 
Cada turma de Educação a Distância reúne características e particularidades próprias. É preciso estabelecer um perfil inicial da turma, seus interesses, suas motivações e habilidades logo no início do curso, para que se possa planejar melhor o curso e promover ajustes que proporcionem uma diminuição da evasão e uma maior aprendizagem [Shih et al. 2007].

Este trabalho apresenta os resultados de uma pesquisa desenvolvida com alunos iniciantes de um curso de graduação em Administração Pública na modalidade EAD para determinar o perfil da turma e no final cruzar essas informações com o desempenho dos alunos na disciplina. Os resultados apontam fatores relevantes que devem ser considerados também por gestores, professores e tutores, para seu aperfeiçoamento e melhores condições de êxito dos alunos no restante do curso.

Além desta introdução, o artigo contempla mais quatro seções. Na segunda seção é feita uma análise de trabalhos para fundamentar as análises e discussões apresentadas. Na seção três define-se o percurso metodológico, com a descrição do experimento, seus procedimentos, a coleta e tratamento dos dados e hipóteses da investigação. Na quarta seção são apresentados os resultados do estudo, com a respectiva análise descritiva. Por fim, na seção cinco são apresentadas as considerações finais e sugestões de trabalhos futuros.

\section{Fundamentação Teórica}

Os principais elementos da EAD e as dificuldades de quem começa a utilizar a modalidade para sua formação e aspectos relacionados com o desempenho dos alunos são descritas nesta fundamentação teórica.

\subsection{Elementos da Educação a Distância}

Os diversos modelos de $\mathrm{EAD}$ diferem em vários aspectos, tais como os tipos de tecnologias empregadas, o controle do ritmo e o local da instrução. Em alguns modelos, os docentes e a instituição possuem um controle completo ou parcial sobre esses. Em outros, o controle recai sobre os alunos, nos quais cada um pode determinar seu ritmo de estudo e de aprendizagem [Ramos 2006].

Como um dos pilares da EAD, os Ambientes Virtuais de Aprendizagem trazem novas perspectivas para o processo de ensino aprendizagem, o que requer dos docentes um olhar inovador, não apenas na forma como se entende a educação, mas principalmente na forma como ocorre a prática, garantindo uma verdadeira mudança de paradigma nesta modalidade educativa, indo além do tradicionalismo a que se encontra interligada [Araújo e Panerai 2012]. O uso desses ambientes pode ser estendido a diversas plataformas e dispositivos, tais como tablets e smartphones, conforme descrito em Voss et al. (2013).

Em Desai et al. (2009) é reforçado que um dos maiores desafios da aprendizagem para instituições e instrutores ao projetarem e implementarem cursos online é proporcionar um senso de comunidade com feedback construtivo e fornecer interações cada vez próximas, bem como promover os sentimentos de amizade, coesão e satisfação entre os alunos. Outro aspecto importante relatado é que a interação bidirecional é uma característica crítica do processo educativo.

Alguns tipos de interações online e práticas instrucionais que podem promover experiências positivas dessa modalidade de ensino-aprendizagem são detalhados em Boling et al. (2012), onde também são listados alguns dos desafios e armadilhas 
potenciais que podem surgir quando indivíduos estão ensinando e/ou aprendendo nesses ambientes virtuais.

\subsection{Barreiras à prática da EAD}

A EAD baseada nas Tecnologias de Informação e Comunicação (TIC) requer uma alfabetização tecnológica que pode se tornar um obstáculo insuperável para alguns; o manejo do editor de textos, planilha de cálculos, instalação de softwares, navegação e pesquisa na Internet, uso do e-mail, são algumas das habilidades que o aluno precisa desenvolver frente às TIC para estudo online. Na EAD os alunos experimentam uma série de dificuldades e necessidades por não contarem com a experiência prévia, nem as habilidades necessárias para enfrentar com êxito esta modalidade de ensino.

De acordo com Mercado (2007), uma das grandes dificuldades dos alunos no primeiro contato com a EAD é a habilidade na utilização de recursos computacionais que são necessários para o dia a dia desta modalidade de ensino. A inabilidade em lidar com as TIC cria dificuldades em acompanhar as atividades propostas, como receber e enviar e-mail, participar de chats, de grupos de discussão, visitar links sugeridos.

No seu trabalho de mestrado, Rabello (2007) organizou os diversos tipos de dificuldades de alunos da modalidade segundo duas categorias:

I. Dificuldades pessoais: referem-se à dificuldade de adaptação à metodologia de educação a distância, tais como: estranhamento ao método EAD, dificuldades de gerenciamento da aprendizagem, a falta de conhecimentos tecnológicos prévios, dificuldades com os conteúdos teóricos excessivos entre outros.

II. Dificuldades com o desenho instrucional: referem-se às dificuldades relacionadas ao modelo instrucional do curso, muitas vezes apresentadas sob forma de crítica, tais como: tutorias, recursos instrucionais, materiais didáticos, plataforma de ensino etc.

Já Ramos (2013), a partir de estudos dos fatores afetivo-sociais predominantes nos alunos de EAD destacados por Moore \& Kearsley (2007), agrupou as dificuldades nas seguintes categorias:

I. Cognitivas: relacionadas com interpretação e compreensão do conteúdo, concentração, abstração etc.;

II. Pedagógicas: ajuda insuficiente no polo, material didático inadequado, conteúdo não proporcional ao tempo etc.;

III. Pessoais: cansaço, organização do tempo, família, motivação, dificuldades com tecnologia entre outras.

A literatura também aponta várias dificuldades relacionadas com a EAD que também são vivenciadas pelos professores, como em Mahdizadeh (2008) e Morais et al. (2013).

\subsection{Desempenho do aluno na EAD}

Em razão da natureza e das particularidades inerentes à $E A D$, os fatores que podem influenciar o desempenho dos alunos nessa modalidade envolvem novas variáveis que não são observadas no ensino presencial: conhecimento tecnológico prévio, tempo de acesso no ambiente virtual, apoio de tutorias, material didático do curso entre outros. 
O desempenho do aluno na EAD pode ser avaliado através de diversas formas, de maneira tradicional ou não: trabalhos escritos, depoimentos, testes, encontros presenciais, monografias, exercícios de aplicação, auto avaliação. A forma de avaliação a ser adotada dependerá do tipo de curso, do público-alvo e da(s) tecnologia(s) empregada(s) [Ramos 2006].

Souza et al. (2012) apresentam uma análise da influência de vários fatores no desempenho de alunos de um curso de aperfeiçoamento a distância, particularmente nas disciplinas de Língua Portuguesa e de Informática. Esses alunos também atuam como docentes do ensino público fundamental e médio. Segundo os autores, variáveis como: sexo, faixa etária, área de formação do aluno e conhecimentos prévios em Língua Portuguesa e Informática não tiveram nenhuma influência no desempenho dos alunos nas disciplinas. Já o nível de escolaridade, o tempo de magistério e o local de residência (capital ou interior) influenciaram no desempenho na disciplina de informática, mas não influenciaram na de Língua Portuguesa.

O ensino a distância tem como principal ferramenta a informática, de maneira que aqueles alunos com menor conhecimento em tecnologia da informação encontram uma barreira que reflete no seu desempenho acadêmico [Chaves \& Andreoli, 2013]. Esses autores avaliaram o impacto do conhecimento em informática no desempenho acadêmico dos alunos de EAD em uma Faculdade e os resultados do trabalho indicaram que há uma correlação significativa entre o desempenho acadêmico dos alunos (nota) e seu conhecimento em informática, ou seja, quanto maior o domínio da informática, melhores são as notas obtidas por estes alunos no curso a distância.

A avaliação de desempenho discente em um AVA foi feita por Souza et al. (2013), a partir de uma análise de múltiplas variáveis, onde o objeto do estudo foi a estrutura curricular de um curso semipresencial. Os resultados apontaram que a organização das disciplinas na matriz curricular do curso favoreceu o desempenho dos alunos no curso.

Outra importante vertente na pesquisa do desempenho de estudantes na EAD é a que trata do estabelecimento de análises e modelos de previsão de desempenho dos alunos, utilizando de forma cada vez mais consistente a Mineração de Dados Educacionais (EDM, do inglês Educational Data Mining). A partir de um conjunto relevante de dados obtidos, por exemplo, nas diversas interações e $\log s$ de acesso dos alunos nos ambientes virtuais, é possível descrever um modelo que permite medir ou prever desempenho como também apontar as possibilidades de evasão de alunos em um curso online. Essas técnicas e métodos já estão consolidados internacionalmente como descritos em Baker \& Yacef (2009); Romero \& Ventura (2013); Peña-Ayala (2014), e nacionalmente, como os trabalhos de Pimentel \& Omar (2006), Santos \& Camargo (2012), Rodrigues et al. (2013), Gottardo et al. (2012, 2014). Segundo Baker et al. (2011), a EDM apresenta oportunidades interessantes para pesquisadores e profissionais da educação, estatística e computação no Brasil.

Assim, como avaliar fatores que podem influenciar no desempenho de alunos iniciantes em curso online e como essa identificação pode impactar em um melhor planejamento e execução do curso? Esta pesquisa buscou esclarecer essas questões, especificamente em uma disciplina introdutória de um curso por EAD, a partir do desenvolvimento do seu método. 


\section{Realização do Experimento}

\subsection{Objetivo}

O objetivo deste experimento foi buscar evidências dos principais fatores que afetam o desempenho de alunos iniciantes em um curso de graduação na modalidade a distância.

\subsection{Participantes}

Participaram do estudo um total de 200 alunos matriculados em uma disciplina ofertada totalmente por EAD. Os alunos residem próximos a 06 polos, a saber: Juazeiro-BA, Capim Grosso-BA, Petrolina-PE, Ouricuri-PE, Salgueiro-PE e Trindade-PE. Foi aplicado questionário no qual os alunos responderam a uma série de perguntas que buscavam estabelecer perfis e as expectativas deles em relação ao curso, à disciplina e à modalidade a distância. A aplicação dos questionários ocorreu no próprio ambiente virtual onde o curso foi desenvolvido, como forma de estimulá-los a interagir no próprio ambiente de ensino em uso no curso.

O perfil dos alunos aponta alguns indicadores que mostram uma heterogeneidade da turma. Em relação à sua formação anterior a este curso, verificou-se que $42,14 \%$ dos estudantes estavam tendo o primeiro contato com o ensino superior e $30 \%$ já tinha iniciado um curso superior anteriormente, mas não concluiu e $27,87 \%$ já tinham um curso superior e/ou pós-graduação. Outra variável importante para a pesquisa foi a experiência com Educação a Distância, verificou-se que 59,71\% estavam tendo a primeira experiência com EAD.

\subsection{Procedimentos}

A realização do experimento deu-se através da disciplina de Introdução à Educação a Distância (IEaD) no Bacharelado em Administração Pública na modalidade a distância ofertado pela Universidade Federal do Vale do São Francisco (UNIVASF), a partir das diretrizes do Programa Nacional de Formação em Administração Pública (PNAP) que teve, além dos desafios já inerentes à própria disciplina introdutória em EAD, o pioneirismo de ser a disciplina inicial da primeira graduação totalmente por EAD ofertada pela UNIVASF.

A disciplina Introdução à Educação a Distância foi toda ofertada pelo ambiente Moodle da Universidade Federal do Vale do São Francisco (UNIVASF) dividida em quatro tópicos distribuídos ao longo de quatro semanas de duração. A estratégia de ensino do conteúdo foi baseada em vídeo-aulas, apostilas, fóruns de discussão, chats e troca de mensagens pelo ambiente. Além do professor, oito tutores online e seis tutores presenciais nos polos apoiaram o desenvolvimento das atividades da disciplina.

Ao longo de cada semana do curso, o material didático foi disponibilizado, no formato de textos, apresentações e vídeo-aulas. Em cada semana, um fórum específico para tratar do conteúdo visto era criado para proporcionar interações entre professor, alunos e tutores.

A realização de um chat com os alunos por polo também ajudou a esclarecer dúvidas sobre conteúdos da disciplina, sobre a modalidade e o curso de Administração Pública. Além disso, proporcionou uma oportunidade de contato com a ferramenta de comunicação síncrona.

$\mathrm{Na}$ penúltima semana do curso, os alunos puderam realizar uma avaliação simulada online, com perguntas do tipo múltipla escolha, preenchimento de lacunas, 
associação de termos e verdadeiro/falso. Essa atividade permitiu uma primeira experiência de avaliação interativa desenvolvida no próprio Moodle.

Os fóruns foram disponibilizados em cada tópico, para comentários e dúvidas e também tiveram diversas postagens pelos alunos e tutores. Fóruns de comentários sobre a avaliação simulada e sobre a avaliação dos alunos também foram bastante utilizados pelos alunos.

A avaliação da disciplina, nos moldes da avaliação simulada anterior, ocorreu logo após a conclusão do conteúdo e para os que não conseguiram aprovação por média maior ou igual a 7,0, foi dada então uma nova chance como avaliação final, onde a maioria conseguiu recuperar a nota e ser aprovado.

Ao final da disciplina, 161 alunos foram aprovados, 15 reprovados por nota e 24 reprovados por não realizarem as atividades avaliativas.

\subsection{Coleta e tratamento dos dados}

A coleta de dados foi feita no próprio ambiente virtual, a partir de um questionário aplicado na primeira semana da disciplina. Nesta coleta, 140 alunos responderam ao questionário, o que representou $70 \%$ dos alunos matriculados no curso.

O Moodle dispõe da ferramenta "Pesquisa" que pode ser facilmente configurada para realizar pesquisas e enquetes com diferentes tipos de questões. A permite exportar os dados coletados em formato de planilha, facilitando análises mais aprofundadas através da utilização de ferramentas estatísticas e de mineração de dados.

No questionário foram feitas seis perguntas sobre o perfil de cada aluno e dezesseis perguntas sobre suas expectativas e habilidades do aluno em relação a temas de interesse da pesquisa. Para este trabalho foram analisadas as respostas de cinco questões que apresentavam indicativos de fatores que poderiam afetar o desempenho do aluno na disciplina. As demais questões foram analisadas e apresentadas em outro trabalho científico cuja análise teve como foco as expectativas e habilidades de alunos iniciantes em EAD em dois momentos do curso [Ramos et al. 2014].

Para possibilitar a realização da aplicação de testes de qui-quadrado foi necessário fazer um pré-processamento dos dados, através de uma agregação de classes, transformando as variáveis explicativas em variáveis com duas classes e possibilitando a construção de tabelas de contingências do tipo $2 \times 2$.

\section{Resultados e Discussões}

Os resultados obtidos na construção deste experimento foram realizados no software estatístico $\mathrm{R}^{1}$, a partir dos dados pré-processados nas etapas anteriores.

\subsection{Tabelas de contingência com aplicação de testes estatísticos}

Durante as análises realizadas neste trabalho utilizamos a técnica de testes inferenciais de Qui-quadrado. O teste utilizado comparou as frequências observadas com as esperadas em cada categoria. Em alguns casos foi necessário utilizar o teste exato de Fisher, quando as tabelas de contingência tiveram valores de caselas inferiores a cinco. Através deste teste foi possível realizar uma análise da relação significativa entre as

\footnotetext{
${ }^{1}$ http://www.r-project.org/
} 
variáveis em estudo com o desempenho dos alunos no curso. A Tabela 01 mostra os resultados dos testes com cinco variáveis escolhidas.

Tabela 01. Tabelas de contingências entre as variáveis e o desempenho.

\begin{tabular}{|c|c|c|c|c|}
\hline \multirow{2}{*}{$\begin{array}{l}\text { [V1] Em relação à sua formação anterior a este } \\
\text { curso: }\end{array}$} & \multicolumn{2}{|c|}{ Desempenho no Curso } & \multirow{2}{*}{ Total } & \multirow{2}{*}{$\begin{array}{l}\text { p valor } \\
\text { (teste) }\end{array}$} \\
\hline & REPROV & APROV & & \\
\hline $\begin{array}{l}\text { É a Primeira Graduação ou Possui Graduação } \\
\text { incompleta anterior }\end{array}$ & 14 & 87 & 101 & 0,9092 \\
\hline Uma ou mais graduação ou já com especialização & 5 & 34 & 39 & $\begin{array}{l}\text { Qui Qu } \\
\text { de Pear: }\end{array}$ \\
\hline \multirow{2}{*}{$\begin{array}{l}\text { [V2] Em relação à sua experiência com Educação } \\
\text { a Distância: }\end{array}$} & \multicolumn{2}{|c|}{ Desempenho no Curso } & \multirow{2}{*}{ Total } & \multirow{2}{*}{$\begin{array}{c}\text { p valor } \\
\text { (teste) }\end{array}$} \\
\hline & REPROV & APROV & & \\
\hline Esse é o meu primeiro curso a distância. & 17 & 70 & 87 & \\
\hline $\begin{array}{l}\text { Já fiz outro(s) curso(s) a distância, com ou sem uso } \\
\text { de ambiente virtual de ensino. }\end{array}$ & 2 & 51 & 53 & $\begin{array}{r}\mathbf{E x} \\
\mathbf{F}\end{array}$ \\
\hline \multirow{2}{*}{$\begin{array}{l}\text { [V3] Meu conhecimento atual em informática é } \\
\text { suficiente para acompanhar a disciplina de } \\
\text { Introdução a EAD. }\end{array}$} & \multicolumn{2}{|c|}{ Desempenho no Curso } & \multirow{2}{*}{ Total } & \multirow{2}{*}{$\begin{array}{l}\text { p valor } \\
\text { (teste) }\end{array}$} \\
\hline & REPROV & & & \\
\hline Discordo+ Discordo completamente + Indiferente & 4 & 6 & 10 & \\
\hline Concordo + Concordo Completamente & 15 & 115 & 130 & $\begin{array}{c}\text { Exato de } \\
\text { Fisher }\end{array}$ \\
\hline \multirow{2}{*}{$\begin{array}{l}\text { [V4] Tenho facilidade em lidar com novas } \\
\text { tecnologias de informação e comunicação }\end{array}$} & \multicolumn{2}{|c|}{ Desempenho no Curso } & \multirow{2}{*}{ Total } & \multirow{2}{*}{$\begin{array}{l}\text { p valor } \\
\text { (teste) }\end{array}$} \\
\hline & REPROV & APROV & & \\
\hline Discordo+ Discordo completamente + Indiferente & 5 & 11 & 16 & $\mathbf{0 , 0 2 8 2 *}$ \\
\hline Concordo + Concordo Completamente & 14 & 110 & 124 & $\begin{array}{l}\text { Qui Qu } \\
\text { de Pear }\end{array}$ \\
\hline \multirow{2}{*}{$\begin{array}{l}\text { [V5] Acredito que os recursos e atividades do } \\
\text { ambiente virtual serão suficientes para a } \\
\text { realização deste curso. }\end{array}$} & \multicolumn{2}{|c|}{ Desempenho no Curso } & \multirow{2}{*}{ Total } & \multirow{2}{*}{$\begin{array}{l}\text { p valor } \\
\text { (teste) }\end{array}$} \\
\hline & REPROV & APROV & & \\
\hline Discordo+ Discordo completamente + Indiferente & 11 & 45 & 56 & \\
\hline Concordo + Concordo Completamente & 8 & 76 & 84 & $\begin{array}{l}\text { Qui Quad } \\
\text { de Pearson }\end{array}$ \\
\hline
\end{tabular}

*Teste com relação significativa.

Através dos resultados encontrados foi possível fazer inferências a respeito da relação existente entre as variáveis em estudo com o desempenho dos alunos.

Em relação a variável [V1] que trata da formação anterior do aluno em cursos de graduação, obtivemos um ( $p$-valor 0,9092). Isso mostra que não existiu uma relação significativa entre o fato de o aluno já ter uma formação superior anterior com o desempenho do mesmo durante o curso.

Por outro lado, pôde-se verificar que a variável [V2] teve uma relação significativa com um ( $p$-valor $\left.0,0096^{*}\right)$. Esta variável, que trata sobre a experiência do aluno com a educação a distância, teve uma relação significativa com o desempenho do aluno. Nesse caso, a dificuldade pessoal de adaptação com a EAD, como afirmado por Rabello (2007) e discutida na seção 2 deste trabalho é atenuada em razão da experiência anterior com a modalidade, o que se comprova com o teste aplicado.

Em relação a variável [V3] que trata se o conhecimento atual em informática (declarado pelo próprio aluno) era suficiente para acompanhar a disciplina de Introdução a EAD. Em relação ao desempenho, esta foi outra variável que teve uma relação significativa com um ( $p$-valor $0,0304^{*}$ ), ou seja, através deste teste foi possível afirmar que alunos que tem conhecimento de informática suficiente para acompanhar a disciplina 
tendem a ter um aumento no desempenho final do curso. Este fato comprova também a afirmação de Rabello (2007) onde o mesmo conclui que a falta de conhecimentos tecnológicos prévios, é um dos fatores que contribuem para o baixo desempenho dos alunos que participam de cursos na modalidade de EAD.

Outra variável que teve uma relação significativa com o desempenho do aluno foi a variável [V4], que mostra a facilidade dos alunos em lidar com novas tecnologias de informação e comunicação. Esta variável teve um ( $p$-valor de 0,0282*) o que demonstra, de acordo com Mercado (2007), que uma das grandes dificuldades dos alunos no primeiro contato com a EAD é a habilidade na utilização de recursos computacionais que são necessários para o dia-a-dia desta modalidade de ensino, incluindo os recursos presentes nos ambientes virtuais (fóruns, chats, tarefas online etc.) assim como o uso de outras plataformas para interagir com o ambiente. Podemos afirmar que o aluno que dispõe destas habilidades tem uma forte relação com um desempenho satisfatório no decorrer do curso.

Por fim foi realizado o teste em relação a variável [V5] onde foi perguntado se os recursos e atividades do ambiente virtual serão suficientes para a realização deste curso. Esta variável em relação ao desempenho do aluno, teve um ( $p$-valor 0,144$)$, de onde podemos concluir que não existiu uma relação significativa entre o fato de o aluno achar que os recursos do ambiente são suficientes com o seu desempenho final.

Dentre as cinco variáveis que foram aplicados os testes de relação, três delas demonstraram relações significativas com o desempenho final do aluno, que foram: [V2], [V3] e [V4]. Todas trataram de variáveis relacionadas com conhecimentos em EAD, Informática e TICs.

\section{Considerações finais e trabalhos futuros}

Este estudo teve como objetivo buscar evidências da influência de algumas variáveis no desempenho acadêmico de alunos iniciantes em um curso de graduação na modalidade a distância.

Verificou-se que uma das grandes dificuldades dos alunos no primeiro contato com a modalidade EAD é relativa à habilidade na utilização de recursos tecnológicos que são necessários para o dia a dia desta modalidade. Para atenuar esse problema, um bom planejamento do curso, aliado a profissionais e procedimentos adequados são necessários.

Através dos experimentos e utilizando tabelas de contingências e testes inferenciais, especificamente os Testes Qui-quadrado e Exato de Fisher, pôde-se identificar em cinco variáveis analisadas quais delas tiveram influência no desempenho dos alunos na disciplina introdutória do curso. Os testes apontaram que experiência prévia com $\mathrm{EAD}$, conhecimento satisfatório em informática e facilidade de adaptação a novas tecnologias tiveram relação significativa com o desempenho do aluno na disciplina online.

Em trabalhos futuros, serão cruzados mais dados dos questionários aplicados com os de desempenho acadêmico e também com os logs dos alunos no ambiente virtual, buscando outras relações com demais variáveis coletadas e o resultado do aluno na disciplina. 
Sugere-se também a mineração dos textos das respostas abertas e dos fóruns da disciplina, na busca de evidências que justifiquem alguns dados e/ou indiquem novas evidências a serem investigadas.

Novos questionários deverão ser aplicados aos mesmos alunos, periodicamente, com a finalidade de fazer o acompanhamento do seu desenvolvimento e também apontar melhores práticas para as próximas turmas do curso. Outro aspecto será a replicação desta pesquisa em diferentes turmas com o intuito de ter uma maior abrangência nas afirmações feitas com os resultados desta pesquisa.

\section{Agradecimentos}

Os autores agradecem o apoio da Universidade Federal do Vale do São Francisco (UNIVASF), por meio da Pró-Reitoria de Pós-Graduação, Pesquisa e Inovação (Edital 18/2013), e da Secretaria de Educação a Distância (SEAD); aos alunos do Curso de Administração Pública. O Professor Alex Sandro Gomes é bolsista DT Nível 2 pelo CNPq apoiado pelos processos no 310466/2012-1 e no 475634/2013-6.

\section{Referências}

Araújo, R., Panerai, T. Relato de Experiência de Blended Learning: O Moodle e o Facebook como Ambientes de Extensão da Sala de Aula Presencial. In Anais do Workshop de Informática na Escola (Vol. 1, No. 1). (2012).

Baker, R. S. J. \& Yacef, K. The state of educational data mining in 2009: A review and future visions. Journal of Educational Data Mining, 1(1), 3-17. (2009).

Baker, R.S.J., Isotani, S., \& Carvalho, A. Mineração de dados educacionais: Oportunidades para o Brasil. Revista Brasileira de Informática na Educação, 19(02), 3. (2011).

Boling, E. C., Hough, M., Krinsky, H., Saleem, H., \& Stevens, M. Cutting the distance in distance education: Perspectives on what promotes positive, online learning experiences. The Internet and Higher Education,15(2), 118-126. (2012).

Chaves, E. D. P., \& Andreoli, C. R. Qual o impacto do conhecimento de informática no desempenho acadêmico dos alunos de EaD?. Revista de Administração IMED, 3(2). (2013).

Desai, M., Hart, J., \& Richards, T. E-learning: Paradigm shift in education. Education, 129 (2), (2009).

Gottardo, E., Kaestner, C. A. A., \& Noronha, R. V. Estimativa de Desempenho Acadêmico de Estudantes: Análise da Aplicação de Técnicas de Mineração de Dados em Cursos a Distância. Revista Brasileira de Informática na Educação, 22(01), 45. (2014).

Gottardo, E., Kaestner, C., Noronha, R.V. Previsão de Desempenho de Estudantes em Cursos EAD Utilizando Mineração de Dados: uma Estratégia Baseada em Séries Temporais. In Anais do XXIII Simpósio Brasileiro de Informática na Educação (SBIE 2012. Rio de Janeiro, RJ). (2012)

Mahdizadeh, H., Biemans, H., \& Mulder, M. . Determining factors of the use of e-learning environments by university teachers. Computers \& Education, 51(1), 142-154. (2008).

Mercado, L. P. L., Dificuldades na Educação a Distância online. Congresso Brasileiro de Educação à Distância. (2007).

Moore, M., Kearsley, G., Educação a distância: uma visão integrada. São Paulo: Thomson Learning. (2007). 
Morais, C., Alves, P., \& Miranda, L. . Valorização dos ambientes virtuais de aprendizagem por professores do ensino superior. CISTI'2013 ( $8^{\text {a }}$ Conferência Ibérica de Sistemas e Tecnologias de Informação), Lisboa. (2013).

Peña-Ayala, A. (2014). Educational data mining: A survey and a data mining-based analysis of recent works. Expert systems with applications, 41(4), 1432-1462.

Pimentel, E. P., \& Omar, N. (2006). Descobrindo Conhecimentos em Dados de Avaliação da Aprendizagem com Técnicas de Mineração de Dados. In Anais do Workshop de Informática na Escola (Vol. 1, No. 1).

Rabello, C. R. L., Aprendizagem na Educação a Distância - dificuldades dos discentes na licenciatura em ciências biológicas na modalidade semipresencial. (Dissertação de Mestrado em Tecnologia Educacional em Ciências da Saúde- Universidade Federal do Rio de Janeiro) (2007)

Ramos, D. K., Perfil dos alunos de Licenciatura a Distância e aspectos que contribuem para aprendizagem. Revista Reflexão e Ação, Santa Cruz do Sul, v.21, n. esp., p.199-220. Disponível em http://online.unisc.br/seer/index.php/reflex (2013).

Ramos, J. L. C. , Rodrigues, R. L., Gomes, A. S., Silva, J. C. S. Análise de expectativas e habilidades discentes na modalidade de ensino a distância. Actas de la $9^{a}$ Conferencia Ibérica de Sistemas y Tecnologías de Informacion. Barcelona-Espanha. v. 1. p. 131-136. (2014).

Ramos, J. L. C. Requisitos para ferramentas de avaliação em ambientes virtuais de ensino (Dissertação de Mestrado em Ciência da Computação-Universidade Federal de Pernambuco). (2006).

Rodrigues, R. L., Medeiros, F. P., Gomes, A. S. Modelo de Regressão Linear aplicado à previsão de desempenho de estudantes em ambiente de aprendizagem. In Anais do XXIV Simpósio Brasileiro de Informática na Educação (SBIE 2013 - Campinas-SP). (2013).

Romero, C., \& Ventura, S. Data mining in education. Wiley Interdisciplinary Reviews: Data Mining and Knowledge Discovery, 3(1), 12-27. (2013).

Santos, H., Camargo, F., \& Camargo, S. Minerando Dados de Ambientes Virtuais de Aprendizagem para Predição de Desempenho de Estudantes. Conferência LACLO, 3(1). (2012).

Shih, T. K., Hung, J. C., \& Jin, Q. A survey of distance education challenges and technologies. International journal of distance education technologies, 11, 1-20, (2007).

Souza, A. A. D., Avelar, E. A., Xavier, D. O., Gatto, R. A., \& Lara, A. L. Análise do Desempenho de Alunos em um Curso Oferecido na Modalidade a Distância. XII Colóquio Internacional de Gestión Universitaria. Veracruz - MEX. (2012).

Souza, T. I., Franco, A. O., Silva, T. E., \& Vasconcelos, H. L. Avaliando o Desempenho Discente em um AVA: Um Estudo de Caso Utilizando Estatística Multivariada. In Anais dos Workshops do Congresso Brasileiro de Informática na Educação (Vol. 1, No. 1).

Voss, G. B., Nunes, F. B., Herpich F. \& Medina, R. D. Ambientes Virtuais de Aprendizagem e Ambientes Imersivos: um estudo de caso utilizando tecnologias de computação móvel. XXIV Simpósio Brasileiro de Informática na Educação (SBIE 2013 Campinas-SP). 